EPJ Web of Conferences 60, 06003 (2013)

DOI: $10.1051 /$ epjconf/20136006003

(C) Owned by the authors, published by EDP Sciences, 2013

\title{
Hadron spectroscopy and exotic states
}

\author{
A. Augusto Alves Jr. ${ }^{1, a}$ on behalf of the LHCb collaboration and presenting results from CMS and ALICE collabora- \\ tions. \\ ${ }^{1}$ INFN and Università di Roma "Sapienza", Rome, Italy
}

Abstract. This contribution summarizes some of the main results from the LHC experiments on hadron spectroscopy and exotic states.

\section{Introduction}

According to our current understanding, the forces responsible to bind quarks into hadrons are described by the non-Abelian field theory called Quantum Chromodynamics (QCD). In QCD-motivated quark potential models, the quarkonia states are described as a quark-antiquark pair bound by an interquark force with a short- distance behavior that is approximately Coulombic, plus an increasing confining potential that dominates at large separations. In one of the simplest approaches, the energy levels can be determined by solving the corresponding nonrelativistic Schrodinger equation in order to obtain the expected masses of the charmonium spectrum, characterized by the radial quantum number and the relative orbital angular momentum between the quark and the antiquark. In particular, all predicted states lying under the DD mass threshold have been observed[1-4].

On the other hand, the possible existence of more sophisticated states than mesons and baryons, like the multiquark states, hybrid mesons and mesonic molecules has been discussed since the early days of the quark model[2, 5-8].

In the last decade, considerable experimental evidence has been collected about the existence of new states, lying in the charmonium mass range, but not fitting well the charmonium mass spectrum picture[9-15]. Most of the observations also suggested that these candidates may be exotic. These studies have been performed at Babar and Belle, two experiments which took data at the $\mathrm{e}^{+} \mathrm{e}^{-}$Beauty Factories at SLAC (Stanford Linear Accelerator Center, USA) and KEK (High-Energy Accelerator Research Organization, Japan), respectively. Confirmations have also come from the CDF experiment, collecting data from $\mathrm{p} \overline{\mathrm{p}}$ interactions at Fermilab,USA.

In this report the main results from the LHC experiments on exotic states candidates are summarized. Section 2 is dedicated to the discussion of the measurements of the $\mathrm{X}(3872)$ mass and cross-section and quantum num-

\footnotetext{
a e-mail: aalvesju@cern.ch
}

bers in the LHCb and CMS experiments. In Section 3, the results of the search for the $\mathrm{X}(4140)$ and $\mathrm{X}(4274)$ states in $\mathrm{B}^{+} \rightarrow \mathrm{K}^{+} \mathrm{J} / \psi \phi$ decays at $\mathrm{LHCb}$ and CMS are presented. In the Section 4, the results from ALICE on search for $\mathrm{H}$-Dibaryon candidates is discussed. The conclusions are presented in Section 5.

\section{Studies on $\mathrm{X}(3872)$}

The $\mathrm{X}(3872)$ resonance was discovered in 2003 by the Belle collaboration in the $\mathrm{B}^{+} \rightarrow \mathrm{K}^{+} \mathrm{X}(3872), \mathrm{X}(3872) \rightarrow$ $\mathrm{J} / \psi \pi^{+} \pi^{-}$decay chain [16]. Its existence was confirmed

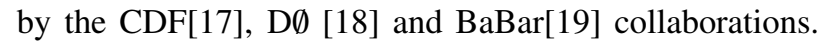
The $\mathrm{X}(3872)$ mass is currently known with $<1.0 \mathrm{MeV} / c^{2}$ precision and the dipion mass spectrum in the decay $\mathrm{X}(3872) \rightarrow \mathrm{J} / \psi \pi^{+} \pi^{-}[20,21]$ has been precisely studied. The $\mathrm{X}(3872)$ quantum numbers was recently measured by $\mathrm{LHCb}$ as $J^{P C}=1^{++}$.

However, despite the cumulated experimental and theoretical effort, the nature of the $\mathrm{X}(3872)$ remains uncertain. Among the possible interpretations for this state currently discussed in the literature, one can remark the mesonic molecule, the hybrid meson and the tetraquark hypotesis. The conventional charmonium interpretation is strongly unfavored.

\subsection{Mass measurements at $\mathrm{LHCb}$}

In $\mathrm{LHCb}$ the analysis is performed a $34.7 \mathrm{pb}^{-1}$ integrated luminosity dataset collected in 2010 in pp collisions at $\sqrt{s}$ $=7 \mathrm{TeV}$. The X(3872) signal has been isolated applying tight cuts in order to reduce the combinatorial background, generated when a correctly reconstructed $\mathrm{J} / \psi$ meson is combined with a random $\pi^{+} \pi^{-}$pair from the primary pp interaction. The selection cuts are optimized using reconstructed $\psi(2 S) \rightarrow \mathrm{J} / \psi \pi^{+} \pi^{-}$decays. The background shape is determined reconstructing "samesign pion" candidates satisfying the same criteria as used for the $\mathrm{X}(3872)$ and $\psi(2 S)$ selection. A further background suppression is reached applying the requirement $\mathrm{Q}$ 


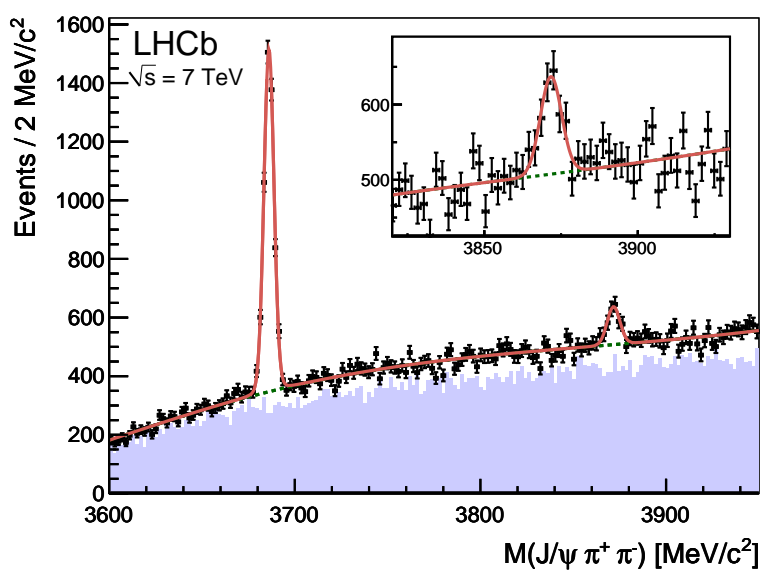

Figure 1. The $M\left(\mathrm{~J} / \psi \pi^{+} \pi^{-}\right)$mass spectrum. The shadowed area represents the "same-sign pion" candidates satisfying the same criteria as used for the $\mathrm{X}(3872)$ and $\psi(2 S)$ selection.The $\psi(2 S)$ and $\mathrm{X}(3872)$ signals are described with a non-relativistic BreitWigner function convolved with a Gaussian resolution function.

$<300 \mathrm{MeV} / c^{2}$, where $Q=\mathrm{M}_{\mu^{+} \mu^{-} \pi^{+} \pi^{-}}-\mathrm{M}_{\mu^{+} \mu^{-}}-\mathrm{M}_{\pi^{+} \pi^{-}}$. See [22] for a detailed discussion on the selection procedure. The masses of the $\psi(2 S)$ and $\mathrm{X}(3872)$ mesons are determined from an extended unbinned maximum likelihood fit of the reconstructed $\mathrm{J} / \psi \pi^{+} \pi^{-}$mass in the interval $3.60<\mathrm{M}_{\mathrm{J} / \psi \pi^{+} \pi^{-}}<3.95 \mathrm{GeV} / c^{2}$. The $\psi(2 S)$ and $\mathrm{X}(3872)$ signals are described with a non-relativistic Breit-Wigner function convolved with a Gaussian resolution function. The intrinsic width of the $\psi(2 S)$ is fixed to the PDG value and the $\mathrm{X}(3872)$ width is fixed to zero in the nominal fit. The ratio of the mass resolutions for the X(3872) and the $\psi(2 S)$ is fixed to the value $\sigma_{\mathrm{X}(3872)} / \sigma_{\psi(2 S)}=1.31$. The background shape is described by the functional form $\mathrm{f}(\mathrm{M}) \propto\left(\mathrm{M}-\mathrm{M}_{\mathrm{J} / \psi}-2 \mathrm{M}_{\pi}\right)^{\mathrm{c}_{0}} \mathrm{e}^{\left(-\mathrm{c}_{1} \mathrm{M}-\mathrm{c}_{2} \mathrm{M}^{2}\right)}$. The fit result is shown in Figure 1. The result is

$$
\mathrm{M}(X(3872))=3871.95 \pm 0.48(\text { stat }) \pm 0.12(\text { syst }) \mathrm{MeV} / c^{2}
$$

The mass of $\mathrm{X}(3872)$ is a critical parameter for the interpretation of this state as $D^{0} \bar{D}^{0}$-bound state. In this context, it is important to draw the reader's attention to the recent precision measurement of the $D^{0}$ mass, performed by $\mathrm{LHCb}$ [23].

\subsection{Production studies at $\mathrm{LHCb}$}

At LHCb, the same sample used to measure the $\mathrm{X}(3872)$ mass has been used to perform X(3872) production studies. See [22] for detailed discussion about the calibration procedure and the treatment of the different sources of systematic uncertainty. The studies are performed just considering candidates lying inside the fiducial region for the measurement defined by

$$
2.5<y<4.5 \text { and } 5<p_{T}<20 \mathrm{GeV} / c
$$

where $y$ and $p_{T}$ are the rapidity and transverse momentum of the $\mathrm{X}(3872)$. The product of the inclusive production

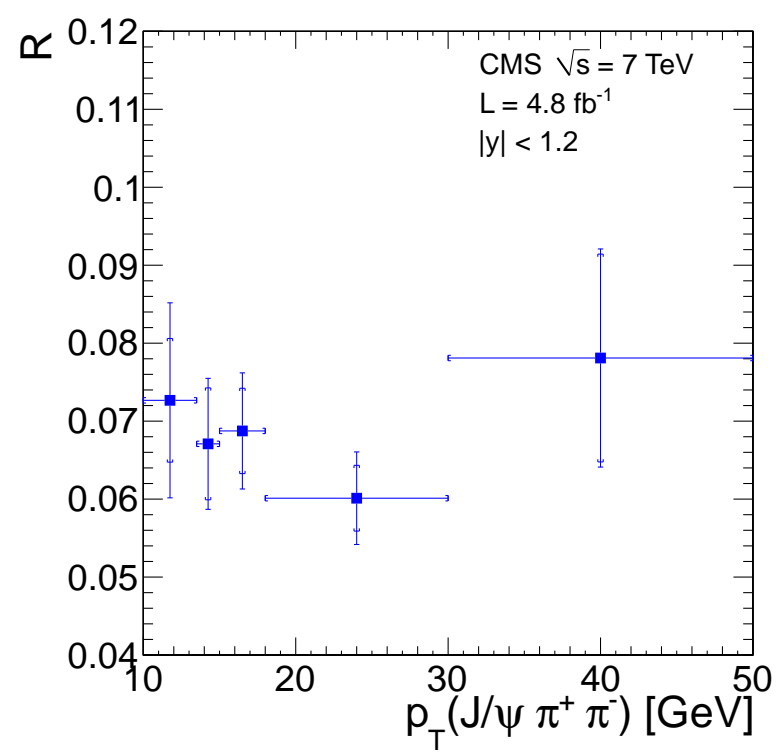

Figure 2. The ratio of the cross section times branching fractions for the $\mathrm{X}(3872)$ and $\psi(2 S)$ in bins of $p_{\mathrm{T}}$ bins as measured by the CMS collabo- ration.

branching fraction of the $\mathrm{X}(3872), \sigma(\mathrm{pp} \rightarrow \mathrm{X}(3872)+$ $\cdots) \times \mathcal{B}\left(\mathrm{X}(3872) \rightarrow \mathrm{J} / \psi \pi^{+} \pi^{-}\right)$, is measured at $\mathrm{LHCb}$ to be $5.4 \pm 1.3$ (stat) \pm 0.8 (syst) nb.

\subsection{Production studies at CMS}

The CMS collaboration studied the $\mathrm{X}(3872) \rightarrow \mathrm{J} / \psi \pi^{+} \pi^{-}$ decay channel using $4.8 \mathrm{fb}^{-1}$ pp collision data collected during 2011 [24]. The measurements are performed for the $\mathrm{X}(3872)$ candidates with a rapidity $\eta<1.2$ and with $10-50 \mathrm{GeV}$ transverse momentum range. The CMS collaboration measured the ratio of the cross section times branching fractions for the $\mathrm{X}(3872)$ and $\psi(2 S)$. The ratio without acceptance corrections for the exclusive $p_{\mathrm{T}}$ bins is shown in Figure 2. The fraction of $\mathrm{X}(3872)$ originating from $\mathrm{B}$ hadron decay in bins of $p_{\mathrm{T}}$ is shown in Figure 3.

The cross section times the branching fractions for the $\mathrm{X}(3872) \rightarrow \mathrm{J} / \psi \pi^{+} \pi^{-}$prompt $\mathrm{X}(3872)$ production, $\sigma^{\text {prompt }}(p p \rightarrow \mathrm{X}(3872)+\cdots) \times \mathcal{B}\left(\mathrm{X}(3872) \rightarrow \mathrm{J} / \psi \pi^{+} \pi^{-}\right)$, has been measured as $1.06 \pm 0.11$ (stat) \pm 0.15 (syst) nb for the $\mathrm{X}(3872)$ candidates with $10-30 \mathrm{GeV} p_{\mathrm{T}}$ range.

Finally, the comparison of the measured dipion mass spectrum of $\mathrm{X}(3872) \rightarrow \mathrm{J} / \psi \pi^{+} \pi^{-}$with simulations where the samples are produced with and without intermediate $\rho^{0}$ decay shows that the $\mathrm{X}(3872) \rightarrow \mathrm{J} / \psi \pi^{+} \pi^{-}$proceed predominately through a $\rho^{0}$ resonance.

\subsection{Quantum number measurements at $\mathrm{LHCb}$}

The LHCb collaboration also measured X(3872) quantum numbers. The measurement is performed using $313 \pm 26$ candidates of the decay chain $\mathrm{B}^{+} \rightarrow \mathrm{K}^{+} \mathrm{X}(3872)$, with $\mathrm{X}(3872) \rightarrow \mathrm{J} / \psi \pi^{+} \pi^{-}$and $\mathrm{J} / \psi \rightarrow \mu^{+} \mu^{-}$selected from $1.1 \mathrm{fb}^{-1}$ dataset collected in 2011 in pp collisions at $\sqrt{s}$ $=7 \mathrm{TeV}$. 


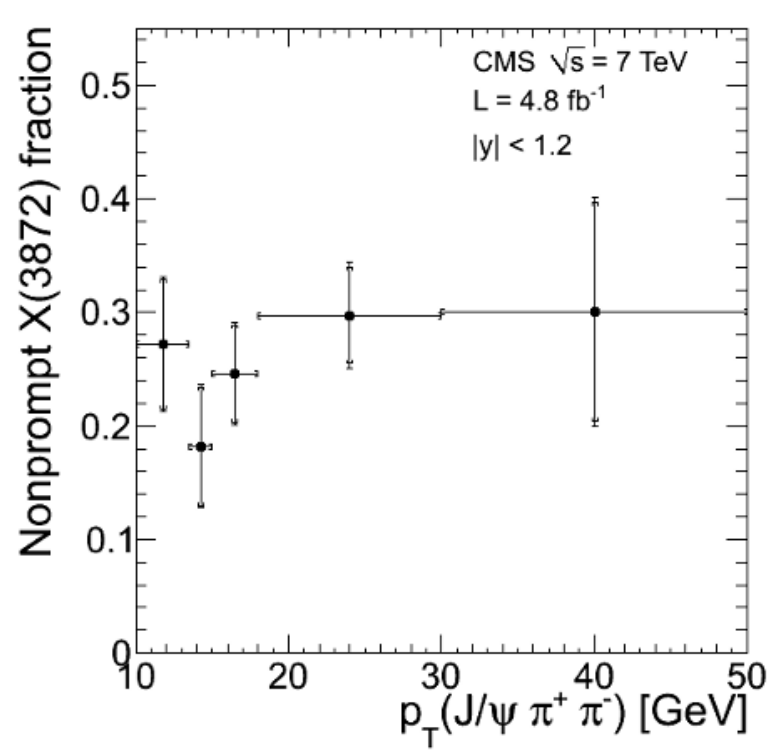

Figure 3. CMS result on the fraction of $\mathrm{X}(3872)$ originating from $\mathrm{B}$ hadron decay in bins of $p_{\mathrm{T}}$.

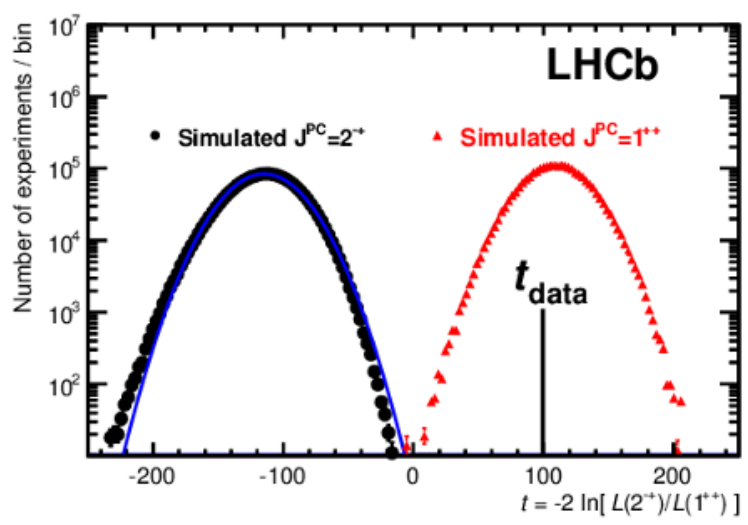

Figure 4. Quantum number measurements at $\mathrm{LHCb}$. The result of the multidimensional likelihood-ratio test favors $J^{P C}=1^{++}$ with more than $8 \sigma$ significance

LHCb makes use of all five angular variables in order to maximize the discrimination power between the hypotheses of $1^{++}$and $2^{-+}$, constrained by the previous experiments. The result of the multidimensional likelihoodratio test is shown in Figure 4 and favors $J^{P C}=1^{++}$with more than $8 \sigma$ significance.

This result rules out the explanation of the $\mathrm{X}(3872)$ meson as a conventional $\eta_{c 2}\left(1^{1} D_{2}\right)$ state. Among the remaining possibilities are the $\chi_{c 1}\left(2^{3} P_{1}\right)$ charmonium, disfavored by the value of the $\mathrm{X}(3872)$ mass, and unconventional explanations such as a $D^{0} \bar{D}^{0 *}$-bound state [25], a tetraquark state [26] or a charmonium-molecule mixture [27].

\section{Search for structures in the $J / \psi \phi$ spectrum}

The CDF collaboration has reported a $3.8 \sigma$ evidence for the $\mathrm{X}(4140) \rightarrow \mathrm{J} / \psi \phi$ state using data collected in proton-antiproton collisions at the Tevatron $(\sqrt{s}=1.96$ $\mathrm{TeV}$ )[28]. In a preliminary update on the analysis [29], the CDF collaboration reported $115 \pm 12 \mathrm{~B}^{+} \rightarrow \mathrm{K}^{+} \mathrm{J} / \psi \phi$ events and $19 \pm 6 \mathrm{X}(4140)$ candidates with a statistical significance of more than $5 \sigma$. The mass and width were determined to be $4143.4_{-3.0}^{+2.9}$ (stat) \pm 0.6 (syst) MeV $/ c^{2}$ and $15.3_{-6.1}^{10.4}$ (stat) \pm 2.5 (syst) $\mathrm{MeV} / c^{2}$, respectively. The relative branching ratio was measured to be $\mathcal{B}\left(\mathrm{B}^{+} \rightarrow\right.$ $\left.\mathrm{K}^{+} \mathrm{X}(4140)\right) \times \mathcal{B}(\mathrm{X}(4140) \rightarrow \mathrm{J} / \psi \phi) / \mathcal{B}\left(\mathrm{B}^{+} \rightarrow \mathrm{K}^{+} \mathrm{J} / \psi \phi\right)=$ $0.149 \pm 0.039$ (stat) \pm 0.024 (syst).

Since a charmonium state at this mass is expected to have much larger width because of open flavor decay channels, the decay rate of the $\mathrm{X}(4140) \rightarrow \mathrm{J} / \psi \phi$ mode, so near to kinematic threshold, should be small and unobservable. Due to these issues, the CDF's report rejuvenated the discussions on exotic hadronic states. It was cogitated that the $\mathrm{X}(4140)$ resonance could be a molecular state [30-32], a tetraquark state $[33,34]$, a hybrid state $[35,36]$ or even a rescattering effect $[15,16]$.

The CDF data also suggested the presence of a second state, referred here as $\mathrm{X}(4274)$ with mass $4274.4_{-6.4}^{+8.4}$ (stat $) \pm$ 1.9 (syst) $\mathrm{MeV} / \mathrm{c}^{2}$ and width $32.3_{-15.3}^{+21.9}$ (stat) \pm 7.6 (syst) $\mathrm{MeV} / c^{2}$. The corresponding event yield was $22 \pm 8$ with $3.1 \sigma$ significance. This observation has also received attention in the literature $[37,38]$. On the other hand, the Belle experiment found no evidence for the X(4140) and $\mathrm{X}(4274)$ states[39, 40].

\subsection{Search for $\mathrm{X}(4140)$ and $\mathrm{X}(4274)$ at LHCb}

The $\mathrm{LHCb}$ analysis[41] starts reconstructing a $\mathrm{B}^{+}$candidate as five-track $\left(\mu^{+} \mu^{-} \mathrm{K}^{+} \mathrm{K}^{-} \mathrm{K}^{+}\right)$vertex using well reconstructed and identified muons and kaons candidates. The $\mathrm{B}^{+}$candidates are required to have $p_{T}>4.0 \mathrm{GeV} / c$ and a decay time of at least $0.25 \mathrm{ps}$. The invariant mass of the $\left(\mu^{+} \mu^{-} \mathrm{K}^{+} \mathrm{K}^{-} \mathrm{K}^{+}\right)$combination is evaluated after the muon pair is constrained to the $\mathrm{J} / \psi$ mass, and all final state particles are constrained to a common vertex. Further background suppression is provided using the likelihood ratio discriminator method.

The $\mathrm{B}^{+} \rightarrow \mathrm{K}^{+} \mathrm{J} / \psi \phi$ invariant mass distribution, with at least one $\mathrm{K}^{+} \mathrm{K}^{-}$combination having an invariant mass within $\pm 15 \mathrm{MeV} / c^{2}$ of the nominal $\phi$ mass was fitted by a Gaussian and a quadratic function resulting in $346 \pm 20 \mathrm{~B}^{+}$ events with a mass resolution of $5.2 \pm 0.3 \mathrm{MeV} / c^{2}$.

The $\mathrm{X}(4140)$ state was searched selecting events within $\pm 15 \mathrm{MeV} / c^{2}$ of the $\phi$ mass. Figure 5 shows the mass difference $M(\mathrm{~J} / \psi \phi)-M(\mathrm{~J} / \psi)$ distribution without $\mathrm{J} / \psi$ or $\phi$ mass constraints. No narrow structure is observed near the threshold. The fit results are $N_{\mathrm{X}(4140)}^{(a)}=6.9 \pm 4.9$ or $N_{\mathrm{X}(4140)}^{(b)}=0.6 \pm 7.1$, depending on the background shape used.

The CDF's fit model was used to quantify the compatibility of the two measurements and considering the $\mathrm{LHCb}$ 


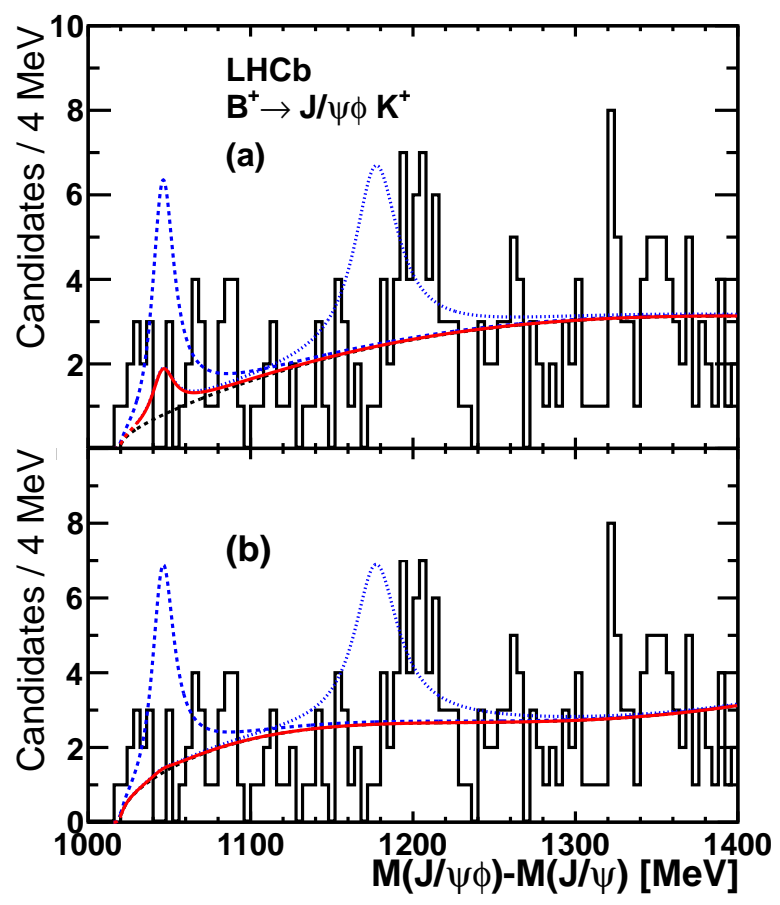

Figure 5. Distribution of the mass difference $M(\mathrm{~J} / \psi \phi)-M(\mathrm{~J} / \psi)$. Fit of the X(4140) signal on top of a smooth background is superimposed (solid red line). The dashed blue (dotted blue) line on top illustrates the expected $\mathrm{X}(4140)$ (X(4274)) signal yield from the CDF measurement. The top and bottom plots differ by the background function (dashed black line) used in the fit: (a) a background efficiency-corrected three-body phase-space; (b) background efficiency-corrected quadratic function.

$\mathrm{B}^{+} \rightarrow \mathrm{K}^{+} \mathrm{J} / \psi \phi$ yield, the efficiency ratio, and the $\mathrm{CDF}$ value for $\mathcal{B}\left(\mathrm{B}^{+} \rightarrow \mathrm{K}^{+} \mathrm{X}(4140)\right) / \mathcal{B}\left(\mathrm{B}^{+} \rightarrow \mathrm{K}^{+} \mathrm{J} / \psi \phi\right)$, one concludes that $\mathrm{LHCb}$ should have observed $35 \pm 9 \pm 6$ events, where the first uncertainty is statistical from the $\mathrm{CDF}$ data and the second includes both the $\mathrm{CDF}$ and $\mathrm{LHCb}$ systematic uncertainties. The $\mathrm{LHCb}$ results disagree with the CDF observation by $\sim 2.7 \sigma$. In the case of the $\mathrm{X}(4274)$ candidate, the same procedure predicts that $\mathrm{LHCb}$ should have observed $53 \pm 19$ X(4274) candidates. The final results are the following upper limits at $90 \% \mathrm{CL}$

$$
\begin{aligned}
& \frac{\mathcal{B}\left(\mathrm{B}^{+} \rightarrow \mathrm{K}^{+} \mathrm{X}(4140)\right) \times \mathcal{B}(\mathrm{X}(4140) \rightarrow \mathrm{J} / \psi \phi)}{\mathcal{B}\left(\mathrm{B}^{+} \rightarrow \mathrm{K}^{+} \mathrm{J} / \psi \phi\right)}<0.07 \\
& \frac{\mathcal{B}\left(\mathrm{B}^{+} \rightarrow \mathrm{K}^{+} \mathrm{X}(4274)\right) \times \mathcal{B}(\mathrm{X}(4274) \rightarrow \mathrm{J} / \psi \phi)}{\mathcal{B}\left(\mathrm{B}^{+} \rightarrow \mathrm{K}^{+} \mathrm{J} / \psi \phi\right)}<0.08 .
\end{aligned}
$$

\subsection{Search for structures in the $\mathrm{J} / \psi \phi$ mass spectrum at CMS}

CMS performed an analysis using $5.2 \mathrm{fb}^{-1}$ pp collision LHC data with $7 \mathrm{TeV}$ center of mass energy[42]. CMS searched for structures in the $\mathrm{J} / \psi \phi$ mass spectrum of $\mathrm{B}$ decays $\mathrm{B}^{+} \rightarrow \mathrm{K}^{+} \mathrm{J} / \psi \phi$, with $\mathrm{J} / \psi \rightarrow \mu^{+} \mu^{-}$and $\phi \rightarrow \mathrm{K}^{+} \mathrm{K}^{-}$. The $M\left(\mathrm{~K}^{+} \mathrm{K}^{+} \mathrm{K}^{-} \mathrm{J} / \psi\right)$ invariant mass spectrum of the selected sample contains $2478 \pm 162$ signal candidates and it is shown in Figure 6. The $\delta M=M\left(\mathrm{~K}^{+} \mathrm{K}^{-} \mu^{+} \mu^{-}\right)-$

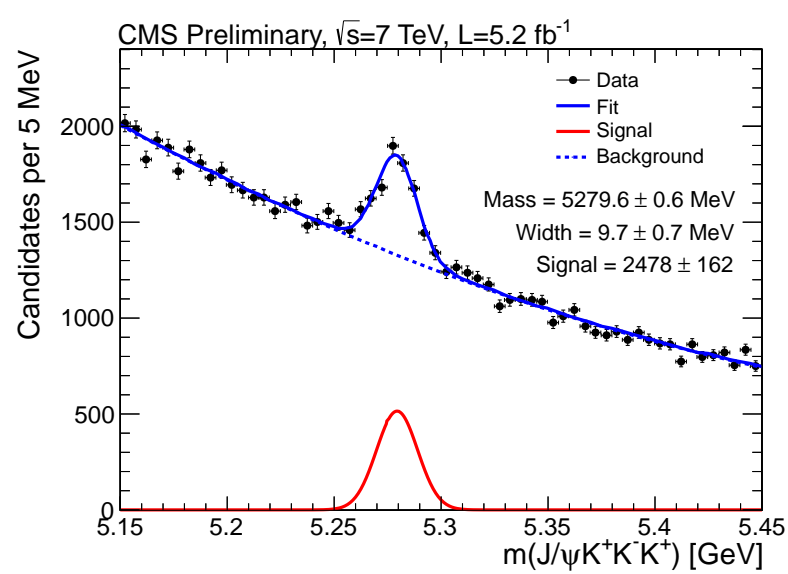

Figure 6. Search for structures in the $J / \psi \phi$ mass spectrum at CMS. The $M\left(\mathrm{~K}^{+} \mathrm{K}^{+} \mathrm{K}^{-} \mathrm{J} / \psi\right)$ invariant mass spectrum of the selected sample.

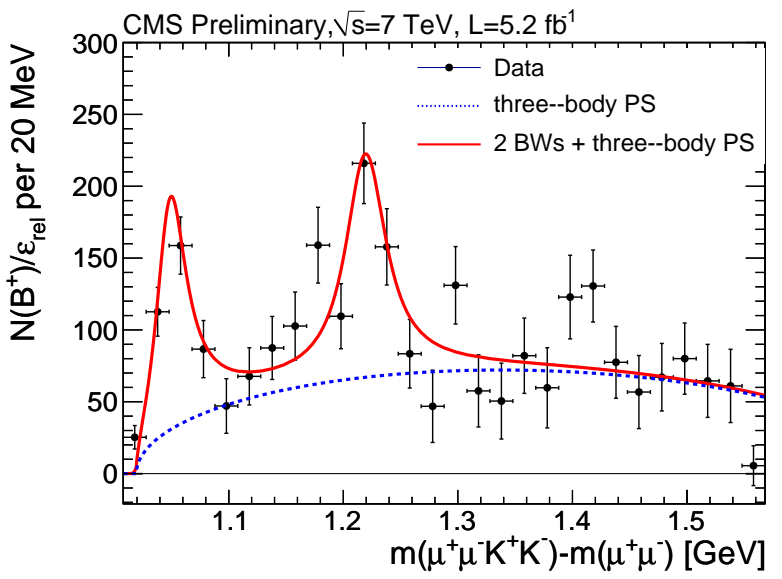

Figure 7. The $\delta M=M\left(\mathrm{~K}^{+} \mathrm{K}^{-} \mu^{+} \mu^{-}\right)-M\left(\mu^{+} \mu^{-}\right)$spectrum obtained from candidates within the $3 \sigma$ of the B mass and after sideband subtraction. CMS interprets the observed structures as the $J / \psi \phi$ resonances with $S$-wave relativistic Breit-Wigner lineshapes over a three-body phase space non-resonant component

$M\left(\mu^{+} \mu^{-}\right)$spectrum obtained from candidates within $3 \sigma$ of the B mass and after sideband subtraction is fitted interpreting the observed structures as the $\mathrm{J} / \psi \phi$ resonances with $\mathrm{S}$-wave relativistic Breit-Wigner line-shapes over a three-body phase space non-resonant component (Figure 7). Taking into account the known $\mathrm{J} / \psi$ mass, CMS reports a structure in the $\mathrm{J} / \psi \phi$ system with mass $m_{1}=4148.2 \pm 2.0$ (stat) \pm 5.2 (syst) $\mathrm{MeV} / c^{2}$ and significance greater than $5 \sigma$. The CMS collaboration, also reports evidence for a second structure with mass $m_{2}=$ $4316.7 \pm 3.0$ (stat) \pm 10.0 (syst) $\mathrm{MeV} / c^{2}$

\section{Search for H-dibaryon at Alice}

Recently, lattice calculations $[9,10]$ have been performed showing evidence for a bound H-Dibaryon. The results of the Alice collaboration search for the H-Dibaryon bound state are based on the analysis of about 13.8 million $\mathrm{Pb}-\mathrm{Pb}$ 


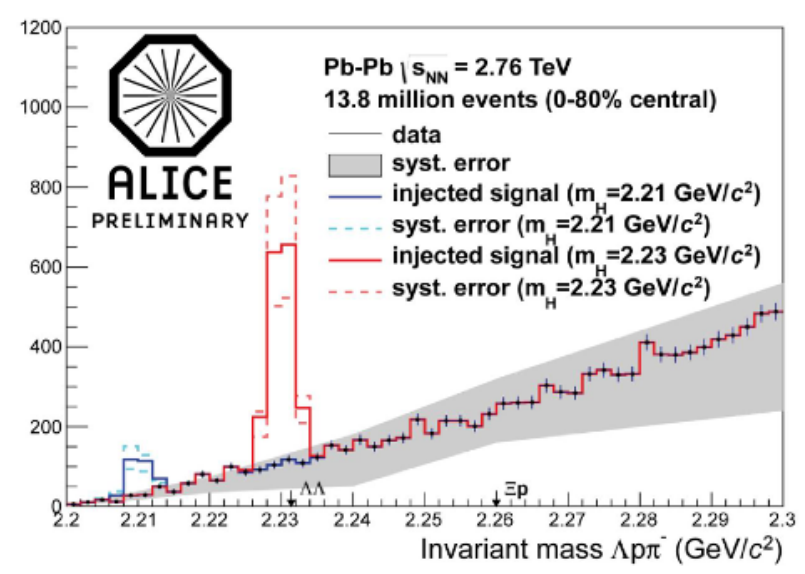

Figure 8. The reconstructed $\mathrm{H}$-dibaryon $(\Lambda \Lambda)$ invariant mass by ALICE.No evidence for a signal of the H-Dibaryon bound state is found.

events in the centrality class of $0-80 \%$ taken with the ALICE apparatus in 2010. The reconstructed invariant mass distribution are shown in Figure 8. No evidence for a signal of the H-Dibaryon bound state is found. Figure 3 also shows the expected signal for the H-Dibaryon for two assumed masses of $2.21 \mathrm{GeV} / c^{2}$ and $2.23 \mathrm{GeV} / c^{2}$ corresponding to binding energies of $21 \mathrm{MeV} / c^{2}$ and $1 \mathrm{MeV} / c^{2}$ respectively. The results are the limits at 99\%CL quoted bellow

- $d N / d y<8.4 \times 10^{-4}$ for $\mathrm{H}$-dibaryon $(\Lambda \Lambda)$ with $2.21 \mathrm{GeV} / c^{2}$,

- $d N / d y<2.0 \times 10^{-4}$ for $\mathrm{H}$-dibaryon $(\Lambda \Lambda)$ with $2.23 \mathrm{GeV} / c^{2}$,

For more details see [43].

\section{Conclusions}

A selection of results on exotic states spectroscopy at the LHC have been summarized. Many new results are expected from the analysis of the 2011 and 2012 datasets and as well as from the new studies currently on-going.

The LHC experiments are in a privileged position to explore the production mechanisms and spectra of the exotic states, delivering competitive results in the heavy flavor sector.

\section{References}

[1] S. Godfrey, S.L. Olsen, Ann.Rev.Nucl.Part.Sci. 58, 51 (2008)

[2] E. Swanson, Int. J. Mod. Phys. A21, 733 (2006)

[3] N. Drenska et al., Riv. Nuovo Cim. 033, 633 (2010)

[4] N. Brambilla, S. Eidelman, B. Heltsley, R. Vogt, G. Bodwin et al., Eur.Phys.J. C71, 1534 (2011)

[5] F.E. Close (2008), arXiv: 0801.2646

[6] J.L. Rosner, J. Phys. Conf. Ser. 69, 012002 (2007)

[7] D. Ebert, R.N. Faustov, V.O. Galkin, Phys. Lett. B634, 214 (2006)
[8] L. Maiani, V. Riquer, F. Piccinini, A.D. Polosa, Phys. Rev. D72, 031502 (2005)

[9] S.K. Choi et al. (BELLE), Phys. Rev. Lett. 100, 142001 (2008)

[10] B. Aubert et al. (BABAR), Phys. Rev. Lett. 95, 142001 (2005)

[11] Q. He et al. (CLEO), Phys. Rev. D74, 091104 (2006)

[12] C.Z. Yuan et al. (Belle), Phys. Rev. Lett. 99, 182004 (2007)

[13] K. Abe et al. (Belle), Phys. Rev. Lett. 98, 082001 (2007)

[14] K. Abe et al. (Belle), Phys. Rev. Lett. 94, 182002 (2005)

[15] S. Uehara et al. (Belle), Phys. Rev. Lett. 96, 082003 (2006)

[16] S. Choi et al. (Belle Collaboration), Phys.Rev.Lett. 91, 262001 (2003)

[17] D. Acosta et al. (CDF Collaboration), Phys.Rev.Lett. 93, 072001 (2004)

[18] V. Abazov et al. (D0 Collaboration), Phys.Rev.Lett. 93, 162002 (2004)

[19] B. Aubert et al. (BABAR Collaboration), Phys.Rev. D71, 071103 (2005)

[20] S.K. Choi et al. (Belle Collaboration), Phys. Rev. D 84, 052004 (2011)

[21] A. Abulencia et al. (CDF Collaboration), Phys.Rev.Lett. 96, 102002 (2006)

[22] R. Aaij et al. (LHCb Collaboration), Eur.Phys.J. C72, 1972 (2012)

[23] R. Aaij et al. (LHCb collaboration), JHEP 1306, 065 (2013)

[24] S. Chatrchyan et al. (CMS Collaboration), JHEP 1304, 154 (2013)

[25] N.A. Törnqvist, Physics Letters B 590, 209 (2004)

[26] L. Maiani, F. Piccinini, A.D. Polosa, V. Riquer, Phys. Rev. D 71, 014028 (2005)

[27] C. Hanhart, Y. Kalashnikova, A. Nefediev, The European Physical Journal A 47, 1 (2011)

[28] Phys. Rev. Lett. 102, 242002 (2009)

[29] T. Aaltonen et al. (CDF Collaboration) (2011), arXiv: 1101.6058

[30] Z.G. Wang, Z.C. Liu, X.H. Zhang, The European Physical Journal C - Particles and Fields 64, 373 (2009), 10.1140/epjc/s10052-009-1156-2

[31] R.M. Albuquerque, M.E. Bracco, M. Nielsen, Physics Letters B 678, 186 (2009)

[32] J.R. Zhang, M.Q. Huang, Journal of Physics G: Nuclear and Particle Physics 37, 025005 (2010)

[33] F. Stancu, Journal of Physics G: Nuclear and Particle Physics 37, 075017 (2010)

[34] N.V. Drenska, R. Faccini, A.D. Polosa, Phys. Rev. D 79, 077502 (2009)

[35] N. Mahajan, Physics Letters B 679, 228 (2009)

[36] Z.G. Wang, The European Physical Journal C - Particles and Fields 63, 115 (2009), 10.1140/epjc/s10052009-1097-9

[37] X. Liu, Physics Letters B 680, 137 (2009) 
[38] S.I. Finazzo, M. Nielsen, X. Liu, Physics Letters B 701, 101 (2011)

[39] C.P. Shen, C.Z. Yuan, H. Aihara, K. Arinstein, T. Aushev, A.M. Bakich, V. Balagura, E. Barberio, A. Bay, K. Belous et al. (Belle Collaboration), Phys. Rev. Lett. 104, 112004 (2010)

[40] J. Brodzicka, Heavy flavour spectroscopy, in Lepton Photon 2009 (LP09) (2010), Vol. DESY-PROC-
2010-04

[41] R. Aaij et al. (LHCb Collaboration), Phys.Rev. D85, 091103 (2012)

[42] E.A. Yetkin, EPJ Web of Conferences 49, 13007 (2013)

[43] B. Dönigus, Nuclear Physics A 904-905, 547c (2013) 